\title{
Medición de la Autoeficacia para la Escritura Académica. Una Revisión Teórico-Bibliográfica
}

\author{
Mauricio González ${ }^{(1)}$, Paulina Meza ${ }^{(2,3) \star}$ y Melissa Castellón ${ }^{(2)}$ \\ (1) Departamento de Psicología, Universidad de La Serena, Avenida Matta №147, Coquimbo, Chile. \\ (e-mail: mgonzale@userena.cl) \\ (2) Departamento de Artes y Letras, Universidad de La Serena, Benavente №1140, La Serena, Chile. \\ (e-mail: pmeza@userena.cl; mcastelloncontreras@gmail.com) \\ (3) Instituto de Investigación Multidisciplinar en Ciencia y Tecnología de la Universidad de La Serena, Avenida Juan \\ Cisternas N¹015, La Serena, Chile.
}

* Autora a quien debe ser dirigida la correspondencia.

Recibido Abr. 16, 2019; Aceptado Jun. 19, 2019; Versión final Jul. 31, 2019, Publicado Dic. 2019

\begin{abstract}
Resumen
Se realiza una revisión de investigaciones publicadas en la literatura nacional e internacional sobre autoeficacia asociada a la competencia para la escritura académica. En especial se analizan aquellas donde se utilizan instrumentos estandarizados de medición para analizar las características principales de esos instrumentos y las conclusiones de los estudios. Se desarrolla un estudio teórico-bibliográfico, cuya muestra quedó constituida por 16 artículos que cumplieron con los criterios de inclusión establecidos. Los resultados centrales indican que el idioma predominante de los instrumentos es el inglés y que los estudios se desarrollan principalmente en Estados Unidos. En Latinoamérica, prácticamente no existen investigaciones sobre el tema. Además, el $62,5 \%$ de los estudios no cumplen con los actuales estándares para la validación de instrumentos. En conclusión, se requiere estudiar de forma más rigurosa la relación entre la autoeficacia para la producción de textos académicos y el desempeño objetivo de los estudiantes en este ámbito, especialmente, considerando textos propios de disciplinas altamente especializadas.
\end{abstract}

\section{Measurement of Self-Efficacy for Academic Writing. A Theoretical-Bibliographic Review}

\begin{abstract}
A review of investigations published in the open national and international literature on self-efficacy associated with the performance of academic writing. Especial consideration is given to those in which standardized measuring instruments are used, for analyzing the main characteristics of such instruments and the conclusions of the corresponding studies. A theoretical-bibliographic research, whose sample consisted of 16 articles that met the inclusion criteria, was developed. The main results show that the English language predominates in the instruments, and that the studies are mainly conducted in the United States. In Latin America practically there is no research published on the subject. In addition, $62.5 \%$ of the investigations does not meet the current standards for instrument validation. In conclusion, it is required to conduct more rigorous research on the relationship between self-efficacy for the production of academic texts and the performance of students in this area, especially considering texts of highly specialized disciplines.
\end{abstract}

Keywords: self-efficacy; academic writing; self-efficacy perception instruments; validation instruments; selfefficacy and academic writing. 


\section{INTRODUCCIÓN}

La escritura es, o debería ser, una actividad central en el currículum universitario, puesto que saber escribir no solo se relaciona con la capacidad de comunicarse adecuadamente, sino que involucra también el desarrollo conjunto de una serie de destrezas cognitivas. Asimismo, la competencia para comunicarse por escrito de manera pertinente a los requerimientos de cada profesión no es fácil de alcanzar y, además, no siempre se enseña de manera explícita durante la formación de pregrado. El logro gradual de esta habilidad se relaciona también con el incremento del logro en las otras materias del currículum de pregrado y con el posterior desempeño profesional. El desafío de incorporar explícita y sistemáticamente el desarrollo de esta competencia en la formación de pregrado requiere de estrategias didácticas y de formas de evaluación adecuadas. Esto tiene la dificultad adicional que los modos de escribir entre diferentes carreras, si bien comparten rasgos similares, tienen características y exigencias particulares acordes con los propósitos y tradiciones de cada una, que se manifiestan en la redacción académica profesional (Staples et al., 2016).

Al respecto, investigaciones recientes han demostrado que la falta de dominio de habilidades de escritura a nivel universitario constituye una de las principales causas de reprobación, retraso y deserción de los estudiantes (Uribe y Carrillo, 2014); y, complementariamente, se ha evidenciado una tendencia al alza en la deserción universitaria en los últimos años (Rodríguez et al., 2018). Además, se ha constatado que una alta proporción de estudiantes universitarios chilenos y extranjeros son malos escritores (Bitrán et al., 2009). Concretamente, se evidencian dificultades en el desarrollo de operaciones cognitivas afines a la producción escrita en áreas del saber especializadas (Tapia et al., 2003; Bitrán et al., 2009; entre muchos otros). Este fenómeno se asocia a la falta de experiencia de los estudiantes en la producción escrita de textos específicos de su área profesional (Tapia et al., 2003), a una formación deficiente y al intento de asumir una postura que vincula la escritura académica con grandilocuencia y oscuridad (Bassi, 2017). Todo ello ha derivado en que se reconozca ampliamente el desarrollo de habilidades de escritura académica como una preocupación educativa sumamente relevante, sobre todo a nivel universitario (Staples et al., 2016).

Una de las variables que se ha relacionado con el desempeño en la escritura es la autoeficacia (Castells et al., 2015; Villalón et al., 2013, entre otros), por lo que existen variados instrumentos que miden la autoeficacia para la escritura (Zimmerman y Bandura, 1994; Pajares, 2007; Büyükikiz et al., 2013, entre muchos otros). En este marco, el constructo de autoeficacia ha demostrado que incide en la predicción del rendimiento y que es el mejor o un muy importante predictor del éxito académico de los estudiantes (Castells et al., 2015; Villalón et al., 2013; Blanco et al., 2016), además de ser un constructo simple y parsimonioso, que lo justifica como una excelente vía para el estudio de las habilidades de escritura. Considerando la dificultad y relevancia de la escritura académica, la importancia del constructo de autoeficacia para el abordaje de la escritura académica y la necesidad de contar con un instrumento válido y confiable que permita medirla de manera pertinente, el objetivo de este estudio es realizar una revisión sistemática de la literatura publicada sobre instrumentos de medición de autoeficacia asociada a la competencia para la escritura académica, a fin de analizar y sintetizar las particularidades de los instrumentos, sus características psicométricas y los principales resultados obtenidos a partir de su aplicación. Tras una revisión sistemática de la literatura científica publicada en diversas bases de datos, motores de búsqueda y redes sociales académicas (WOS, Scielo, EBSCO, ERIC, SAGE, Google Scholar, Research Gate, entre otros), no se encontraron estudios de revisión con este mismo propósito.

\section{OTROS ANTECEDENTES}

Para el desarrollo del trabajo es pertinente analizar algunos aspectos relacionados con la escritura académica, la autoeficacia en relación con la escritura académica y con los instrumentos de medición de la autoeficacia para la escritura.

\section{Escritura académica}

En términos generales, la escritura académica se entiende como la composición de textos escritos por parte de individuos que pertenecen al ámbito académico, y cuya principal audiencia son sujetos miembros de esta misma esfera (Carlino, 2005). En consecuencia, el propósito de este tipo de escritura es académico, lo que implica que explora algunos aspectos del conocimiento y que, generalmente, es producida por estudiantes en distintas áreas disciplinares. A ello se puede agregar que la escritura académica es ampliamente reconocida como una forma elaborada de discurso y una producción gramaticalmente compleja (Staples et al., 2016), dificultad que aumenta si se considera que existen diferencias disciplinares en el tipo de escritura en cuestión. Estas diferencias se advierten en aspectos como organización, vocabulario y convenciones académicas (PakTao, 2008). Por tanto, la escritura académica nunca es uniforme, objetiva ni neutral, ya que lo que hace el escritor es mostrar a los lectores las respuestas plausibles a un determinado problema y, también, su postura respecto a un tema (Badley, 2009). 
La escritura académica es un ejercicio complejo porque no solo requiere decir o transcribir el conocimiento, sino que, más bien, implica un trabajo cognitivo complejo de construir, deconstruir y reconstruir el conocimiento; conectar, desconectar y reconectar conceptos; describir y volver a describir puntos de vista sobre el mundo; así como también formar, deformar y reformar las ideas (Badley, 2009). En esta línea, el tipo de escritura en cuestión tiende a definir modos de discurso o formas de interactuar con el conocimiento de cada disciplina. Para ello, el escritor debe aplicar distintos tipos de conocimiento, tales como: resumir, sintetizar, criticar, persuadir, investigar, citar, parafrasear, adoptar las convenciones de un género, seleccionar palabras y patrones gramaticales más formales y menos personales, entre otros (Tardy, 2010). Todo ello evidencia que la escritura académica es un ejercicio muy complejo, pues el estudiante debe considerar múltiples factores, como: la situación retórica, y, por tanto, conocimiento de su audiencia y de diferentes contextos como el cultural, social y académico; el tipo de información y el género que usará para comunicarse; además, debe plantearse un propósito claro que se encuentre dentro de las expectativas de la comunidad académica a la que pertenece o quiere pertenecer (Irving, 2010). Este carácter complejo de la escritura académica aumenta si se considera que ella es siempre una forma de evaluación que requiere demostrar conocimiento y evidenciar dominio de ciertas habilidades disciplinarias para pensar, interpretar y presentar (Lowe y Zemliansky, 2010).

A partir de lo anterior, a modo de síntesis, se puede señalar que la escritura académica se refiere al complejo proceso de producción de textos en contextos de formación académica, o bien a los productos textuales que ese proceso genera. Estos textos, además, poseen características particulares, dependientes del área disciplinar en la que ellos se producen. Asimismo, considerando la importancia que tiene para la calidad del ejercicio de la profesión y para el propio desarrollo cognitivo del futuro profesional, la escritura académica, tanto como proceso o como texto/producto, debería ser un componente básico de la formación académica de estudiantes universitarios.

\section{Autoeficacia y escritura académica}

El constructo de autoeficacia fue propuesto por Bandura (1977) en su teoría del Aprendizaje Social y se ha convertido en una variable muy relevante a la hora de explicar el desempeño social y académico (Corry y Stella, 2018). La autoeficacia puede definirse como la creencia de un individuo en su capacidad para tener éxito en una situación específica o para realizar competentemente una tarea específica (Bandura, 1977, 1997, 2012; Blanco, 2010, entre otros). De acuerdo con la teoría social cognitiva (Bandura, 1982, 1997), la motivación y la conducta asociada dependen de las cogniciones (creencias) que las personas tienen sobre una situación determinada y sus consecuencias. Bandura $(1977,2006)$ señala que la autoeficacia debe ser estudiada respecto a un dominio de competencias o a una tarea en un contexto específico y concreto ya que una persona podría tener altos niveles de autoeficacia en un dominio y bajo en otro. De acuerdo con la conceptualización de este autor, la autoeficacia se caracteriza por: (a) creencias sobre acciones futuras, no desempeño pasado; (b) creencias sobre capacidades, no expectativas de resultados; (c) especificidad de dominio, no evaluación de rasgos generalizados (Klassen y Klassen, 2018).

A fin de deslindar más claramente el concepto en cuestión, es útil distinguir la autoeficacia de otros constructos como el autoconcepto, que refiere a autopercepciones multidimensionales orientadas al pasado; autoestima, que alude a juicios personales de un individuo sobre su propio valor; locus de control, que apunta a las creencias generalizadas de que las acciones afectan los resultados; y la confianza, ya que esta refleja en sí mismo la fuerza de la creencia (ella es una persona segura de sí misma), pero no el objetivo o dominio específico para esa creencia (Klassen y Klassen, 2018). Por otro lado, la autoeficacia se relaciona estrechamente con la motivación (Boscolo y Hidi, 2017), de manera que, en una situación académica, los alumnos o profesores con una alta autoeficacia tendrían mayor motivación para aprender, lo que se traduciría en un mayor rendimiento académico, el que, a su vez, incrementaría la percepción de autoeficacia (Honicke y Broadbent, 2016). Además, desde una perspectiva teórica, la autoeficacia se puede fortalecer observando el éxito de alguien o por la persuasión social. Adicionalmente, se sabe que está influenciada por el género y la edad (Yokoyama, 2019).

Por lo anterior, el estudio de la autoeficacia en los procesos de escritura es un campo muy relevante y que puede aportar considerablemente al debate sobre los procesos de formación de competencias de escritura a nivel de pregrado (McCarthy et al., 1985; Shell et al., 1989; Zimmerman y Bandura, 1994; Shell et al.,1995; Pajares y Valiante, 2006; Pajares et al., 1999; Pajares et al., 2001; Pajares, 2003, 2007; Wyatt, 2018). Diversos autores han estudiado la autoeficacia en los procesos de escritura, entre quienes cabe destacar a McCarthy et al. (1985), quienes fueron pioneros en establecer la relación entre estos dos constructos. Posteriormente, Zimmerman y Bandura (1994) predijeron, en el contexto de los estudiantes universitarios, que la autoeficacia para regular las actividades de escritura, junto con la autoeficacia para el logro académico, podrían predecir 
el logro de la escritura más allá de la aptitud verbal. A partir de ahí, se han masificados los estudios que abordan esta temática (por ejemplo, Blanco et al., 2016; Honicke y Broadbent, 2016; Golombek et al., 2018, entre otros), por lo que también ha surgido la necesidad de contar con instrumentos de medición pertinentes.

\section{Instrumentos de medición de la autoeficacia para la escritura}

De acuerdo con la revisión de Olivari y Urra (2007), los instrumentos más utilizados para medir la autoeficacia son los de autorreporte (en formato de escala Likert), los cuales pueden ser de dos tipos: unos, focalizados en un dominio específico (autoeficacia académica, social, de autogestión, etc.); y, otros, aquellos que buscan medir la autoeficacia general, en contradicción con lo señalado inicialmente por Bandura $(1993,2006)$, como la escala generalizada de autoeficacia (Luszczynska et al., 2005). El hecho de contar con un instrumento de medición adecuado y pertinente para el estudio de la autoeficacia, focalizada en el estudio de la producción de textos académicos, resulta fundamental para su abordaje científico. Para identificar los instrumentos adecuados, se requiere la revisión de la bibliografía existente, a fin de conocer si existen instrumentos pertinentes que cumplan con los actuales estándares científicos de validez y confiabilidad; y, además, que sea pertinente para evaluar la autoeficacia para la escritura a nivel universitario, lo que implica la capacidad para escribir textos disciplinares con formatos, objetivos y destinatarios muy diferentes, que pueden ir desde ensayos hasta informes técnicos especializados.

\section{MÉTODO}

El objetivo central de este trabajo téorico-bibliográfico fue realizar una revisión de artículos de investigación que reportan la utilización de instrumentos de medición de la autoeficacia asociada a la competencia para la escritura académica, con énfasis en aquellas donde se utilizan instrumentos estandarizados de medición, para analizar las características principales de esos instrumentos y las conclusiones de los estudios. En consecuencia, este estudio corresponde a un diseño de revisión sistemática de la literatura científica relacionada con la medición de la autoeficacia para la escritura de textos académicos y profesionales.

Para desarrollar la investigación se buscaron artículos que cumplieran con algunos de los siguientes criterios de inclusión: a) estudios empíricos, no teóricos; b) propuestas de instrumentos con su respectivo estudio psicométrico; c) trabajos específicos sobre autoeficacia y escritura; d) estudios empíricos en los que se utilizaron instrumentos de autoeficacia para la producción de textos académicos. Esta exploración se realizó en las bases de datos: WOS, SCOPUS, Scielo, ERIC, Science Direct y Google Scholar, bajo las palabras clave: self-efficacy, self-efficacy to writing, writing assesment, writing self-efficacy, autoeficacia, autoeficacia en la escritura, autoeficacia para la escritura académica. El periodo de búsqueda consideró entre los años 2008 a 2018 y, en cuanto a la lengua de las investigaciones, se incluyeron estudios publicados en español, inglés y portugués. Además, se seleccionaron solo artículos empíricos publicados en revistas con evaluación de pares. Tras esta búsqueda, se encontraron, en primera instancia, 20 artículos; sin embargo, se eliminaron 4, pues no correspondían al género Artículo de Investigación o eran versiones no publicadas de un trabajo. Una vez aplicados los criterios de inclusión, obtuvimos, finalmente, 16 artículos para este estudio de revisión. En cuanto a la revisión de los artículos seleccionados, se analizó el contenido de cada uno de ellos a partir de la elaboración de diversas tablas. Luego, la información considerada pertinente y relevante se sintetizó en una sola tabla (Ver Tabla 1).

\section{RESULTADOS Y DISCUSIÓN}

En la Tabla 1 se presenta un resumen con la información principal extraída de los 16 artículos que cumplieron con los criterios de inclusión. Luego, se presenta una síntesis descriptiva de los resultados más relevantes, junto a su respectiva discusión. En la Tabla 1, AFC es Análisis factorial Confirmatorio; AFE es Análisis Factorial Exploratorio; AFE* es Análisis Factorial con SPSS; ACP es Análisis de Componentes Principales; y $\mathrm{CCl}$ es confiabilidad consistencia interna; además, se marca con un asterisco $\left({ }^{*}\right)$ el instrumento principal, que es para el cual se detallan los indicadores de validez y confiabilidad. Luego, para un mejor ordenamiento de los resultados obtenidos y su discusión, ellos se distribuyen en 4 subapartados: a) Caracterización general de los estudios revisados, b) Instrumentos para medir la autoeficacia para la escritura académica, c) Características técnicas y propiedades psicométricas de los instrumentos, d) Resultados relevantes de las investigaciones revisadas. 


\begin{tabular}{|c|c|c|c|c|c|c|c|c|}
\hline & 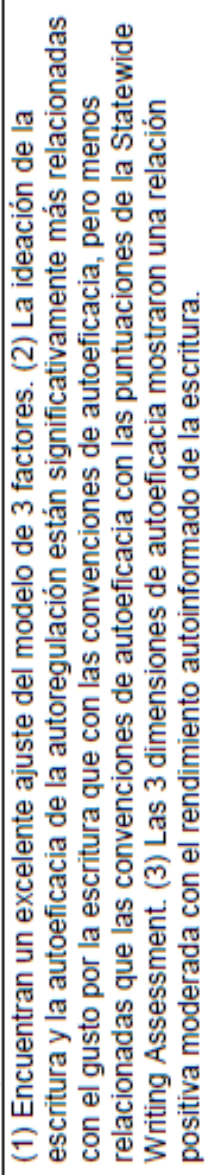 & 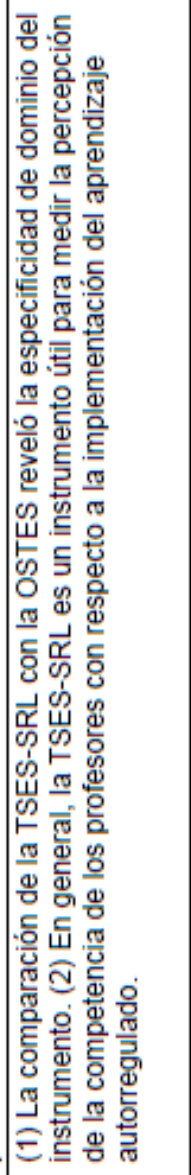 & 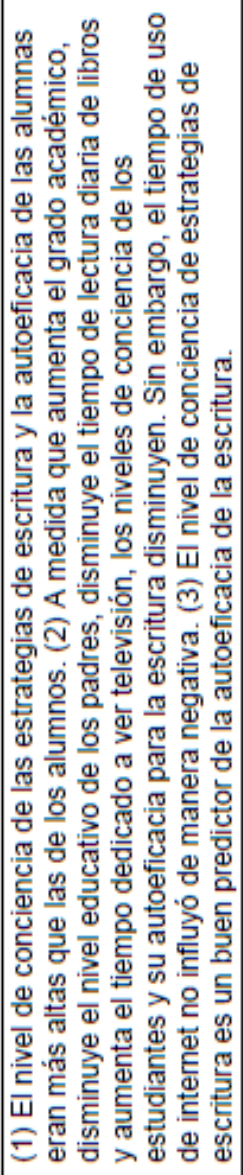 & 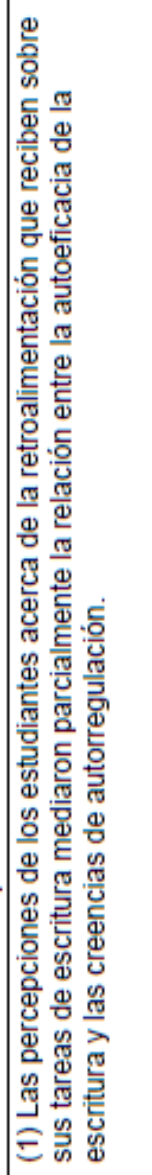 & 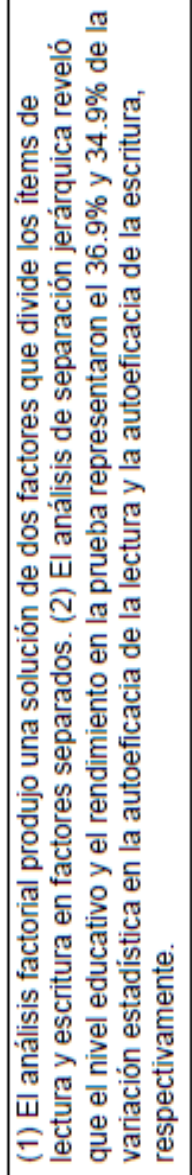 & 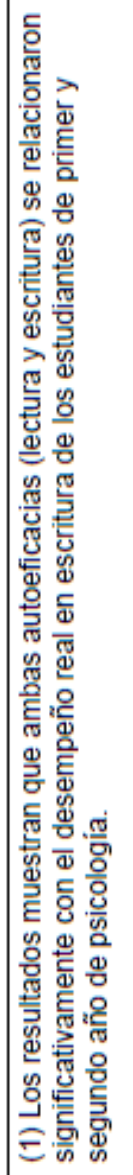 & 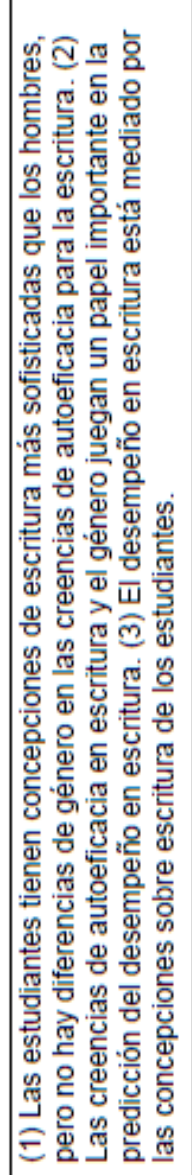 & 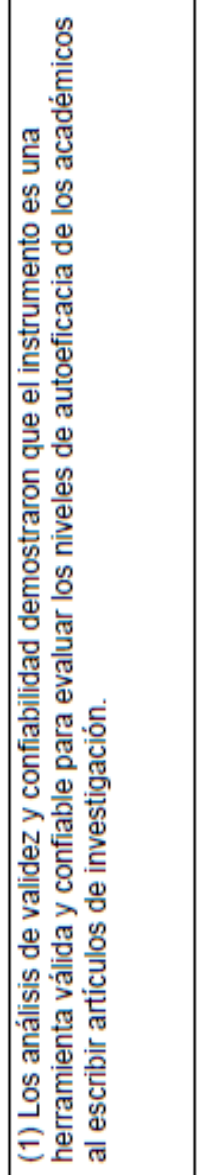 \\
\hline & 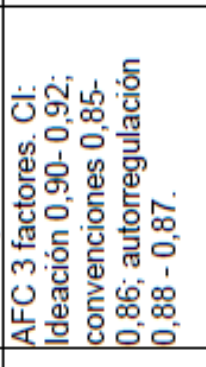 & 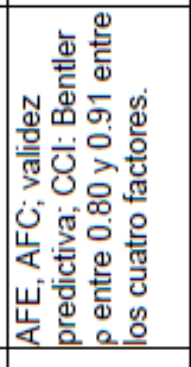 & 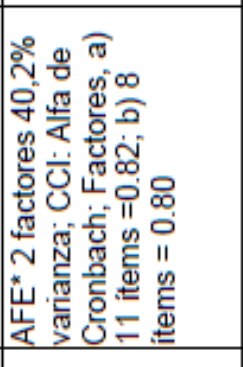 & 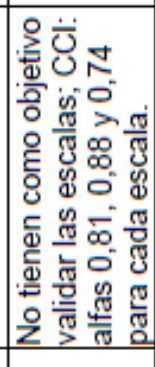 & 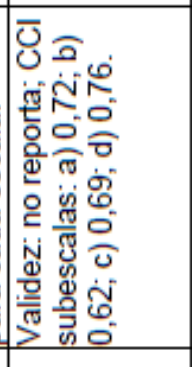 & 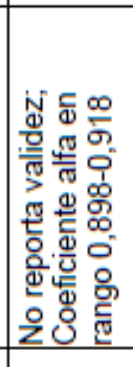 & 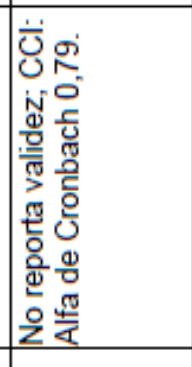 & 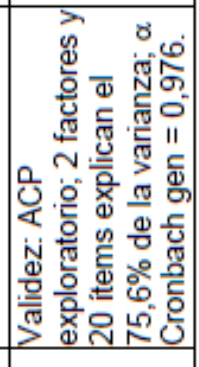 \\
\hline & 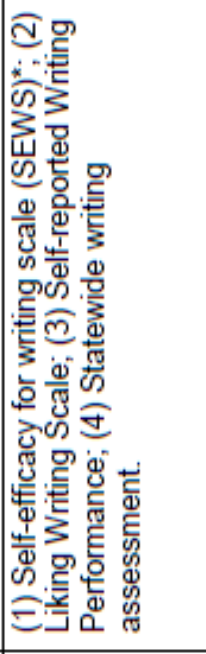 & 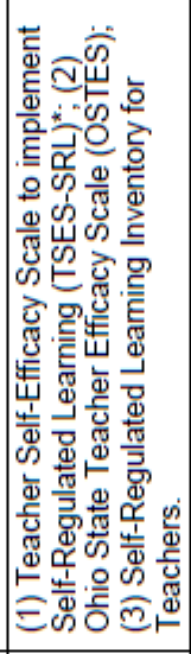 & 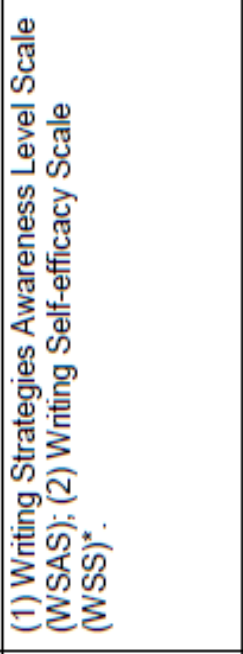 & 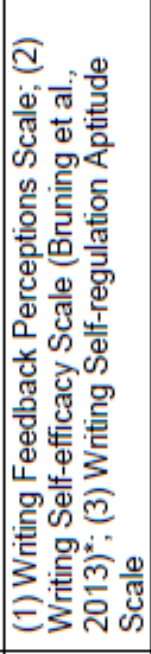 & 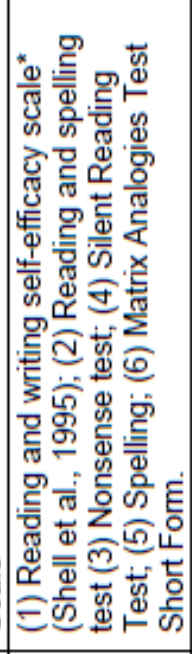 & 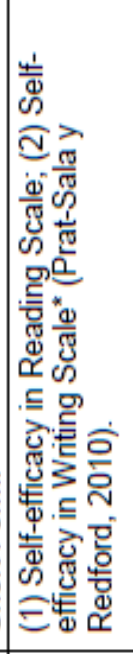 & 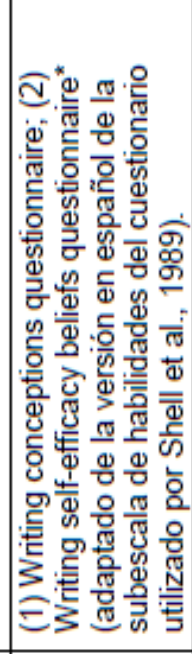 & 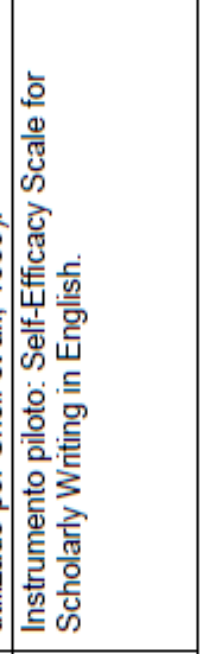 \\
\hline & 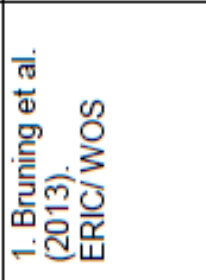 & 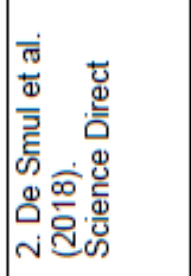 & 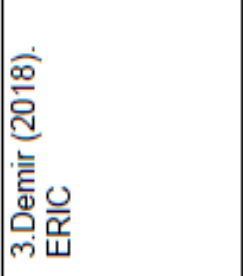 & 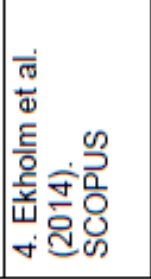 & 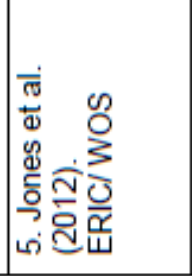 & 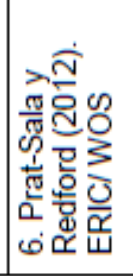 & 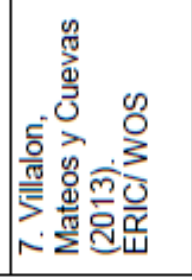 & 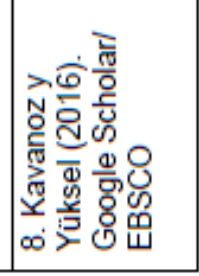 \\
\hline
\end{tabular}




\begin{tabular}{|c|c|c|c|c|c|c|c|c|}
\hline & 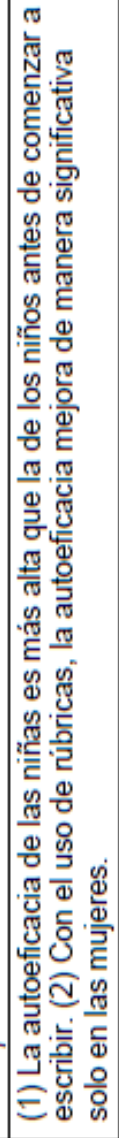 & 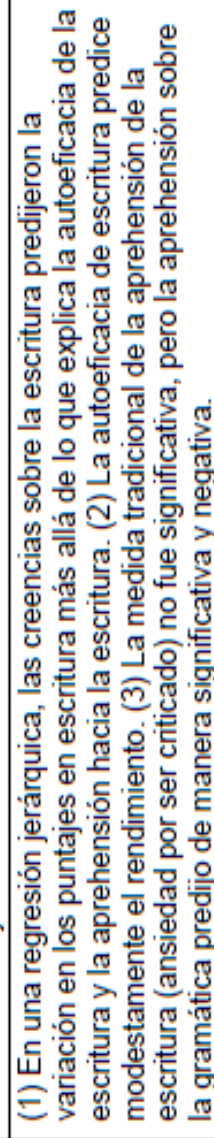 & 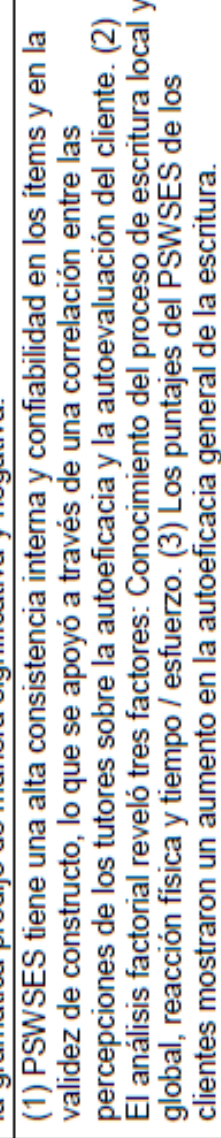 & 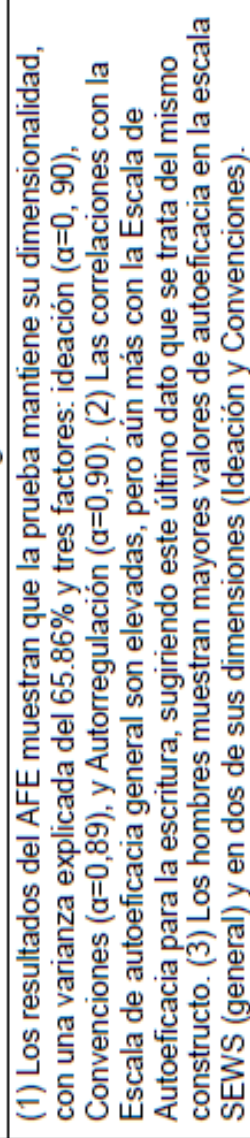 & 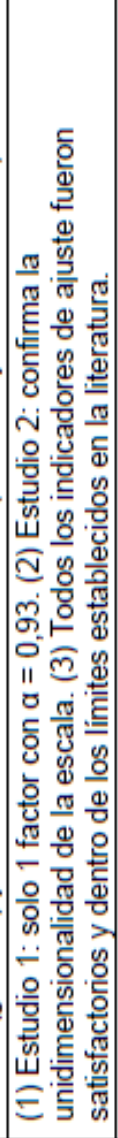 & 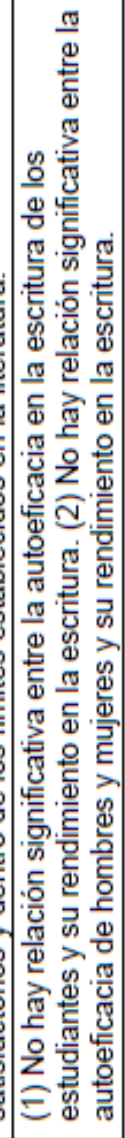 & 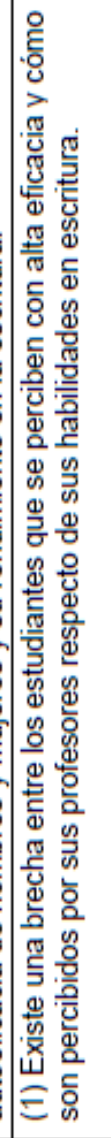 & 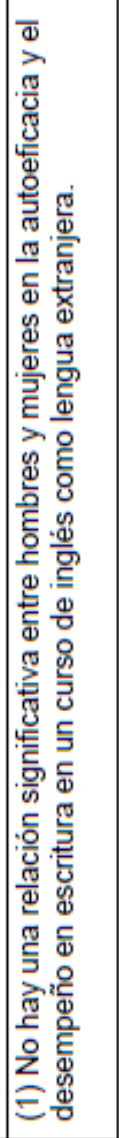 \\
\hline 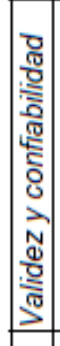 & 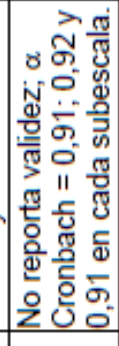 & 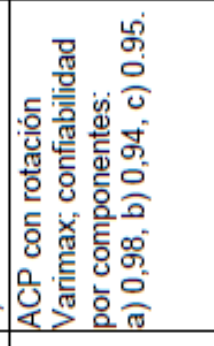 & 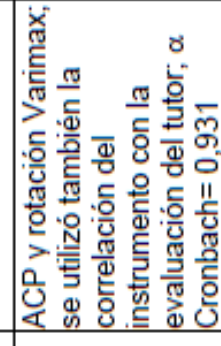 & 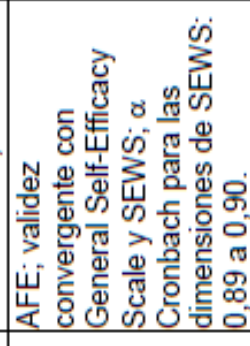 & 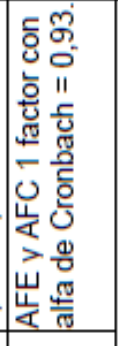 & 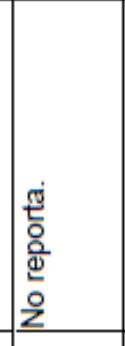 & 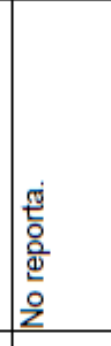 & 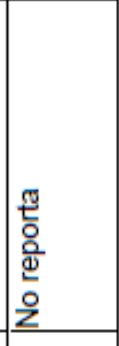 \\
\hline & 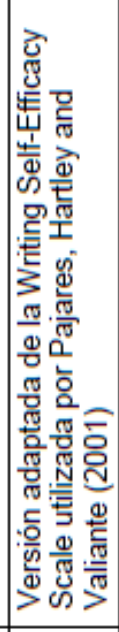 & 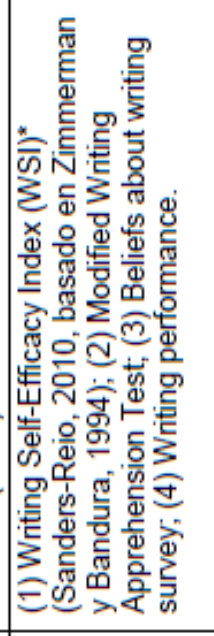 & 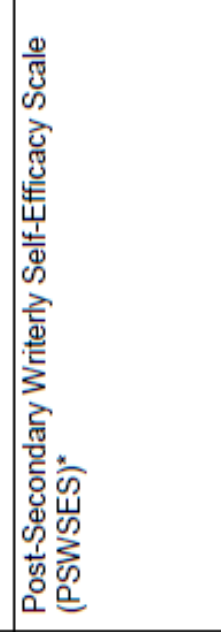 & 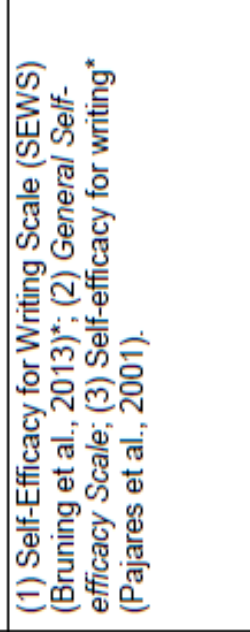 & 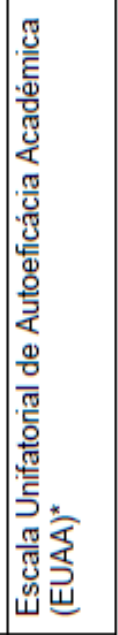 & 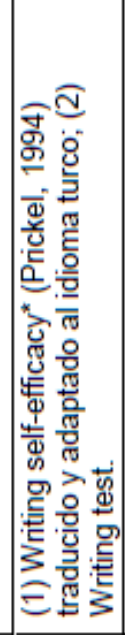 & 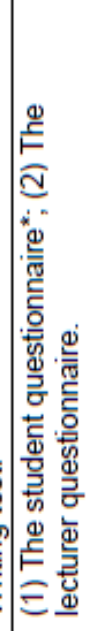 & 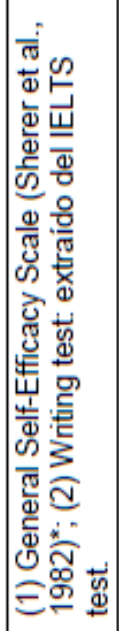 \\
\hline $\mid$ & 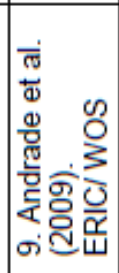 & 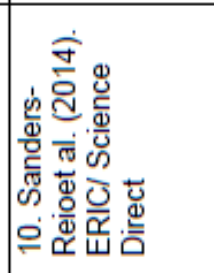 & 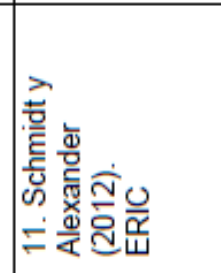 & 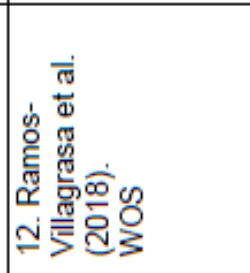 & 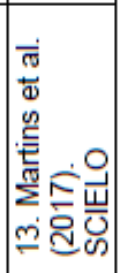 & 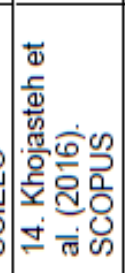 & 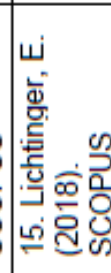 & 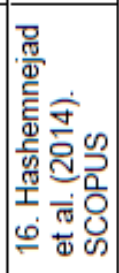 \\
\hline
\end{tabular}




\section{Caracterización general de los estudios revisados}

Los objetivos generales que persiguen las investigaciones de los artículos revisados se pueden dividir en dos grupos. Por un lado, están aquellos trabajos cuyo propósito es diseñar y validar una escala de autoeficacia para la escritura; y, por otro, los que utilizan escalas desarrolladas previamente. En esta investigación, se encontraron 5 trabajos del primer grupo: Ramos-Villagrasa (2018), Bruning et al. (2013), Schmidt y Alexander (2012), Kavanoz y Yüksel (2016), Martins et al. (2017). El resto de las investigaciones aplican un instrumento para estudiar la autoeficacia en relación con otras variables, como, por ejemplo, la percepción de los profesores de sus prácticas docentes (De Smul, 2018), el nivel de conciencia sobre las estrategias de escritura que tienen estudiantes de secundaria (Demir, 2018) y la relación entre la autoeficacia de los estudiantes que se especializan en la enseñanza de inglés como lengua extranjera y su rendimiento en la escritura en hombres y mujeres (Hashemnejad et al., 2014). En esta línea, entonces, la mayoría de las investigaciones tienen como objetivo general evaluar la relación entre autoeficacia y otra variable, mientras que la menor parte de ellas pretenden proponer o validar un instrumento para medir la autoeficacia para la escritura. Entre las variables estudiadas, la más frecuente es el sexo de la muestra, cuyo resultado varía entre investigaciones que afirman que las mujeres poseen mayor autoeficacia (Andrade et al., 2009; Sanders-Reio et al., 2014; entre otras) y otras que señalan que no existen diferencias significativas (Hashemnejad et al., 2014; Khojasteh et al., 2016). No se encontraron resultados que apunten a una mayor percepción de autoeficacia en los hombres. Curiosamente, las investigaciones que reportan la inexistencia de diferencias significativas entre hombres y mujeres fueron realizadas en Irán, por lo que podría sugerir la existencia de la influencia de la cultura en este resultado. Cabe señalar, además, que en una de dichas investigaciones (Hashemnejad et al., 2014) la información presentada respecto del método y mediciones del estudio no permite generalizar con seguridad las conclusiones ofrecidas.

Por otro lado, el análisis revela que, en los 16 artículos estudiados, la distribución geográfica de las muestras utilizadas para investigar la percepción de autoeficacia es variada. Ellas se concentran, principalmente, en: Europa (España, Reino Unido, Bélgica, Noruega), América del Norte (solo Estados Unidos) y Medio Oriente (Irán, Israel, Turquía) con 5 casos en cada uno de estos sectores. La muestra restante se ubica en América del Sur, concretamente, en Brasil. Esto demuestra que en diversos sectores del globo ha habido interés en el estudio de la percepción de autoeficacia en relación con la escritura y, por tanto, se cuenta con datos de sujetos de distintos lugares del mundo, aunque con una concentración importante en Estados Unidos. Ello es concordante con el lugar de origen de las primeras investigaciones sobre autoeficacia, que surgen, precisamente, en este último país con las investigaciones de Bandura (1982, 1997, 2006) y Bandura et al. (1985), quien acuña la idea de que las creencias autorreferentes son un aspecto esencial para comprender el comportamiento humano. Se evidencia, por tanto, la ausencia total de investigaciones y muestras analizadas en torno al tema en Chile y, prácticamente, en toda América Latina.

\section{Instrumentos para medir la autoeficacia para la escritura académica}

De las 16 investigaciones revisadas, 14 utilizaron una escala específica de autoeficacia para la escritura. En la mayoría de ellas, estos instrumentos se aplicaron junto a otro centrado en algún dominio concreto, por ejemplo, autoeficacia académica, para la lectura, de aprendizaje, del desempeño docente, entre otros. En nuestro análisis, el constructo de autoeficacia, por su origen teórico, es más consistente entre los diferentes estudios revisados; por el contrario, en el ámbito de la escritura, existe mayor heterogeneidad en la definición de esos constructos, ya que o bien se considera la habilidad o competencia para escribir en abstracto, o bien se focaliza en algunos aspectos generales como la capacidad de organización, regulación o creatividad. Otros instrumentos encontrados miden aspectos motivacionales, actitudinales o creativos respecto de la escritura 0 se focalizan en las competencias para la lectura o la expresión oral. Además, se encontraron 2 escalas de autoeficacia académica en general y 7 escalas para evaluar algún aspecto específico de la escritura, pero que no estaban asociadas con la autoeficacia. No se encontraron instrumentos que midan la autoeficacia para la producción de textos disciplinares específicos, hecho que evidencia un vacío en este ámbito.

Respecto de los principales instrumentos para medir la competencia para la producción de textos, la revisión de la literatura científica muestra que este constructo se ha asociado sistemáticamente con el constructo de autoeficacia y autorregulación, lo que ha resultado muy adecuado al proporcionar un marco teórico parsimonioso y bien fundamentado respecto al rol motivacional que cumple en el desarrollo de competencias en dominios específicos. Asimismo, la mayoría de los instrumentos miden la autoeficacia para la producción de textos o alguna variante conceptual muy similar. Además, los instrumentos actualmente utilizados se basan, en gran medida, en un instrumento propuesto inicialmente por Zimmerman y Bandura (1994), que consiste en una escala tipo Likert cuyos ítems están elaborados sobre la base de afirmaciones sobre creencias de autoeficacia respecto a la producción de textos académicos generales. 
En cuanto a la eficacia para la producción de textos, por el contrario, no existe la misma claridad conceptual, ya que esta tarea requiere el despliegue de diferentes habilidades y conocimientos, algunos de los cuales son de carácter general, como el dominio de la ortografía y criterios generales de redacción, mientras que otros son muy específicos y propios de la naturaleza del texto a producir y su contexto. Por ejemplo, escribir un estudio de caso, un ensayo o un informe técnico pueden tener estructuras y estilos de redacción muy diferentes y, además, variar entre distintas áreas disciplinares.

Respecto a lo anterior, y considerando lo señalado por Bandura (2006) en cuanto que la autoeficacia debe ser preferentemente estudiada en el marco de desempeños en tareas o contextos específicos, ya que las personas pueden ser eficaces para algunas tareas pero no para otras, la poca especificidad en el uso del concepto producción de textos académicos puede generar una distorsión o una ambigüedad en la interpretación de los puntajes obtenidos en estas mediciones. En la literatura revisada, no se encontraron instrumentos que midan la autoeficacia para la producción de textos académicos disciplinares claramente identificados. Se infiere, entonces, que la elaboración de instrumentos que consideren tanto los aspectos generales como los específicos de la producción de textos podría ser un aporte importante en este campo de estudio. Sobre todo, si consideramos las dificultades y las variaciones existentes en la escritura académica, ya referidas en este trabajo.

Otro aspecto relevante de los instrumentos analizados es que la mayoría de ellos no son nuevas propuestas, sino que constituyen adaptaciones de otros, dentro de los cuales las escalas más utilizadas son la de autoeficacia para la escritura de Bruning et al. (2013), la de autoeficacia para la escritura de Pajares et al. (2001) y la de autoeficacia para la lectura y la escritura de Shell et al. (1989). A su vez, la mayoría de estos instrumentos se basan en Zimmerman y Bandura (1994) y su propuesta de la Escala de eficacia autorregulatoria de la escritura, focalizada en la autorregulación de la escritura de proyectos y procesos (por ejemplo, "puedo empezar a escribir sin dificultad"). En cuanto al idioma de los instrumentos utilizados, ocho de ellos están en inglés ( $50 \%)$, dos en español (12,5\%), dos en persa $(12,5 \%)$, uno en portugués $(6,25 \%)$, uno en noruego $(6,25 \%)$, uno en turco $(6,25 \%)$ y uno en flamenco $(6,25 \%)$. Ello confirma la posición del inglés como lengua franca en el tipo de instrumentos que aquí interesa y, además, evidencia la necesidad de contar con instrumentos como los que se analizan en otras lenguas y para otras culturas.

\section{Características técnicas y propiedades psicométricas de los instrumentos}

Una característica común entre los instrumentos revisados en esta investigación es que todos ellos emplean un formato de escala Likert con una graduación de cinco opciones. Esto es concordante con las sugerencias de diversos expertos, quienes, por una parte, recomiendan grados impares para la configuración de este tipo de escalas (Jackson, 2009; Rubin y Babbie, 2011); y, por otra, sugieren cinco, seis o siete puntos como cantidad adecuada para la mayoría de los constructos (Willits et al., 2016).

Ahora bien, al analizar la cantidad de ítems de los instrumentos de autoeficacia para la escritura seleccionados, se encontraron algunas diferencias entre ellos. Así, las escalas más numerosas son, primero, la presentada en la investigación de Sanders-Reio et al. (2014), que tiene 55 ítems, divididos en tres subescalas: contenido (substantive), autorregulación (self-regulatory) y mecánica (mechanical); y, en segundo lugar, la de Lichtinger (2018) con 40 ítems. Por el contrario, las menos cuantiosas son la presentada por Ramos-Villagrasa et al. (2018), tomada de Pajares et al. (2001), que posee 10 ítems, y la adaptación de Pajares et al. (2001), que elabora Andrade (2009), con 11 ítems. Esta variabilidad podría explicarse por la diversidad de objetivos que se persiguen con la aplicación de los distintos instrumentos, por las variables contempladas para relacionar la percepción de autoeficacia en la escritura y, también, por la complejidad técnica del constructo a medir. En nuestra revisión, las escalas más numerosas fueron aplicadas a universitarios (Sanders-Reio, 2014 et al.; Lichtinger, 2018), mientras que una de las menos numerosas estaba dirigida a estudiantes de escuelas primarias y secundarias.

En cuanto a la formulación de los ítems, lo más recurrente es el uso de oraciones declarativas en primera persona singular, como, por ejemplo, "I can think of many ideas for my writing" o "I can punctuate my sentences correctly" (Bruning et al., 2013). Esta forma de presentar los ítems se condice con el formato de los primeros instrumentos de percepción de autoeficacia (Zimmerman y Bandura, 1994), en los que también se utilizaban oraciones declarativas de primera persona singular, como, por ejemplo, "I can start writing with no difficulty" o "I can find a way to concentrate on my writing even when there are many distractions around me" (Zimmerman y Bandura, 1994:850). Por tanto, la mayoría de los autores de instrumentos de autoeficacia creados han optado por seguir el modelo seminal en la elaboración de sus ítems, ya sea para crear nuevos instrumentos o para realizar las adaptaciones pertinentes para sus investigaciones.

La población meta de la gran mayoría de los instrumentos analizados son estudiantes universitarios (9 de 16, lo que equivale al $56,25 \%)$. Además de eso, encontramos tres trabajos (18,75\%) focalizados en estudiantes 
secundarios (Bruning et al., 2013; Villalón et al., 2013; Demir, 2018); uno (6,25\%) en adultos encarcelados (Jones, 2012); uno (6,25\%) en profesores de primaria (De Smul et al., 2018); uno (6,25\%) en estudiantes de primaria y secundaria (Andrade et al., 2009); y uno (6,25\%) en personal académico de distintas universidades (Kavanoz y Yüksel, 2016). El hecho de que el foco de interés sean los estudiantes de educación superior podría deberse a que, en las últimas décadas, tanto en el ámbito anglosajón como hispanohablante, se han concretado diversas iniciativas en torno a la escritura en la educación superior tendientes a fortalecer la escritura académica, hasta constituir un campo de investigación diverso y en desarrollo (Sabaj, 2009). El diagnóstico y la intervención de la autoeficacia de estudiantes de educación superior sería, entonces, una vía prometedora para ayudar a los universitarios a superar los serios problemas de escritura que presentan y, así, facilitarles el ingreso a la vida académica y a su comunidad disciplinar. Esto, a su vez podría tener un impacto, por ejemplo, en la disminución de la deserción de estudiantes que no logran llegar a dominar las prácticas escriturales generales y menos las específicas de su profesión.

Con respecto a los indicadores de confiabilidad y validez de los instrumentos, siete de los dieciséis estudios no presentaron indicadores de validez (43.75\%) y tres no reportaron confiabilidad (18.75\%), lo que no permite evaluar la calidad psicométrica de esos instrumentos y la calidad de los resultados reportados en esos artículos. Dos trabajos (12,5\%), cuyo objetivo fue la construcción de un instrumento, reportaron tanto Análisis Factorial Exploratorio (AFE) como Confirmatorio (AFC). En un artículo $(6,25 \%)$ se reportó AFC más validez de criterio y en otro $(6,25 \%)$ AFE con validez convergente. Tres artículos $(18,75 \%)$ reportaron Análisis de Componentes Principales (ACP) y uno $(6,25 \%)$ reportó AFE, pero sin indicadores de ajuste. Cuando se utilizó un criterio independiente, fue una puntuación de rendimiento en una tarea de escritura. Por otro lado, el $75 \%$ reportó como indicador de confiabilidad el alfa de Cronbach, un artículo reportó un coeficiente no conocido (Bentler r) y el resto no reportó ningún indicador. Considerando todo lo anterior, el $62,5 \%$ de los trabajos analizados no reportan procedimientos para validar las mediciones o reportan ACP, por lo que no estarían cumpliendo con los actuales estándares para la validación de instrumentos (Lloret et al., 2014; Muñiz y Fonseca-Pedreros, 2019).

Así mismo, respecto a la confiabilidad, la mayoría de los de artículos reporta un indicador de consistencia interna, sin embargo, no señalan si la matriz de datos cumplía con los supuestos relacionados con el carácter ordinal de los ítems, la distribución normal de las respuestas y la cantidad de ítems por subescalas para interpretar este indicador de manera pertinente. La falta de indicadores psicométricos adecuados levanta un manto de incertidumbre sobre la validez de los instrumentos reportados. Es importante considerar también que las medidas de autoeficacia no son equivalentes a las medidas de desempeño real en tareas de escritura (Podsakoff et al., 2012); aunque hay estudios que reportan correlaciones entre ambas medidas, solo corresponde afirmar que las primeras pueden predecir a las segundas (Castells et al., 2015; Villalón et al., 2013). Sin embargo, es necesario indagar más sobre esta relación, puesto que se sabe que las personas menos competentes tienden a percibirse más autoeficaces que las personas objetivamente más competentes (Ramos-Villagrasa et al., 2018), tal como evidencia el denominado efecto Dunning-Kruger (Kruger y Dunning, 1999)".

Este hecho, sin duda, podría conducir a resultados y conclusiones contradictorias que no permiten determinar si los estudiantes con mejor autopercepción también producen mejores textos (Salinas et al., 2017). Lo anterior es avalado por Castells et al. (2015:591), cuando afirma que "es necesario realizar otros estudios que analicen los escritos de los estudiantes en los diferentes perfiles. Solo de ese modo será posible confirmar que lo que los estudiantes declaran hacer está en línea con lo que realmente hacen en una situación real de escritura". Se hace evidente, entonces, la necesidad de realizar estudios que aborden la relación entre la percepción de autoeficacia y el desempeño real en la producción de textos, más aún, se requiere que se repliquen estudios para evaluar la robustez de las teorías en contextos diferentes, tanto desde el punto de vista cultural como del ámbito de desempeño estudiado, utilizando instrumentos con mejores indicadores de validez y confiabilidad.

Por otro lado, la autoeficacia para la escritura ha sido considerada como unidimensional. Posteriormente Pajares (2007) sugirió que podía ser un constructo bidimensional. Así, una dimensión reflejaría habilidades básicas como la ortografía, la puntuación y el uso de verbos; mientras que otra comprendería el uso de habilidades más complejas de composición como la estructuración de párrafos y el uso de oraciones temáticas. Al respecto, Bruning (2013) señala que la actividad de escribir requiere al menos tres tipos de capacidades: la generación de ideas (ideación), la expresión de esas ideas de acuerdo con convenciones del lenguaje (convenciones) y la autogestión de la conducta y la toma de decisiones (autorregulación). Zimmerman y Kitsantas (2007) ponen énfasis en este último aspecto de autorregulación señalando que los escritores planifican, escriben y reflexionan sobre lo que escribieron. 


\section{Resultados relevantes de las investigaciones revisadas}

Entre los estudios analizados, aquellos que se ocuparon de la relación entre variables, esto es, que no propusieron, validaron o evaluaron un instrumento, sino que analizaron la percepción de autoeficacia en relación con distintas variables, se encontraron resultados diversos. Uno de ellos es el de Demir (2018), quien, al estudiar la relación entre el nivel de conciencia sobre las estrategias de escritura y la autoeficacia en escritura, descubrió que tanto el nivel de conciencia de las estrategias de escritura como la autoeficacia eran más altas en las mujeres que en los hombres. Además, observó que los niveles de conciencia de los estudiantes y su autoeficacia en la escritura disminuyen ante las siguientes variables: mayor grado académico, menor nivel educativo de los padres, menor duración de la lectura diaria de libros y mayor tiempo para ver la televisión. Se suma a ello que el tiempo para el uso de internet no se reflejó de manera negativa. Además, en la investigación se determinó que el nivel de conciencia de las estrategias de escritura es un factor predictivo significativo de la autoeficacia en escritura. Otro resultado interesante es el de Lichtinger (2018), quien explora las diferencias existentes entre las creencias de autoeficacia de los estudiantes universitarios con respecto a sus habilidades de redacción y las percepciones que tienen de estas últimas sus profesores. Tras la investigación, Lichtinger (2018) encuentra que existe una brecha importante entre la percepción relativamente fuerte de eficacia que poseen los estudiantes y el nivel más bajo en el que los profesores perciben las habilidades de escritura de sus alumnos.

Otra investigación interesante es la de Jones et al. (2012), quienes examinan la autoeficacia de lectura y escritura en adultos encarcelados. Los resultados sugieren que el nivel educativo y también el rendimiento en las pruebas de velocidad lectora, palabras sin sentido (nonsense words) y ortografía contribuyeron significativamente a la autoeficacia de lectura y escritura, ya que casi el $40 \%$ de la variación en las creencias de los participantes en cuanto a la eficacia de la lectura y la escritura se explicaron por su rendimiento en dichas pruebas. Por último, otro resultado importante de destacar es el de Sanders-Reio et al. (2014), quienes encontraron que las creencias sobre la escritura predijeron la variación en las puntuaciones de escritura mejor de lo que se explica por la autoeficacia y el miedo (apprehension) a la escritura (por ejemplo, el miedo a ser criticado). Sobre la base de este resultado, los autores sugieren que las creencias sobre la escritura pueden ser un punto de apoyo para enseñar a escribir a los estudiantes.

En términos generales, las principales conclusiones de los estudios conducen a afirmar que la autoeficacia para la escritura se relaciona de manera significativa con otras variables, tales como: el nivel de conciencia sobre las estrategias de escritura (Demir, 2018) o el gusto por la escritura (Bruning et al., 2013). A su vez, las investigaciones que han abordado la relación entre el constructo de autoeficacia y las tareas de producción escrita concluyen que, efectivamente, la percepción de la autoeficacia influye en el desempeño de la escritura, estableciendo una relación entre ambas variables (Prat-Sala y Redford, 2012; Bruning, et al., 2013; Villalón et al., 2013). Esta idea es corroborada por otros autores que concuerdan en que la percepción de la autoeficacia es una variable que influye en la predicción del rendimiento, y en que es el mejor o un muy importante predictor del éxito académico de los estudiantes (Castells et al., 2015; Villalón et al., 2013); sin embargo, coinciden también en que la gran mayoría de los trabajos se basan exclusivamente en las declaraciones de los estudiantes sobre su eficacia y no en su desempeño real (Castells et al., 2015).

Ahora bien, en cuanto a los métodos utilizados para abordar la relación entre escritura y percepción de la autoeficacia, en general, se trata de investigaciones descriptivas en las que se analiza una muestra acotada; además, no hay estudios que se focalicen en carreras de pregrado específicas, que, como es sabido, poseen requerimientos de producción escrita diferentes. En este sentido, hasta la fecha, no se cuenta con resultados que apunten a dilucidar la relación entre percepción de autoeficacia y escritura académica disciplinar o profesional. Por otro lado, en una parte importante de los estudios analizados, los sujetos debieron responder una serie de preguntas que apuntaban a sus creencias sobre sus habilidades de escritura, pero sin realizar ninguna tarea de escritura (Bruning et al, 2013, por ejemplo). En muy pocos casos, además de responder un cuestionario, debían realizar alguna actividad de escritura como, por ejemplo, la redacción de un ensayo (Khojasteh et al., 2016). Esto ha tenido como consecuencia el hecho de que contemos con una abundante cantidad de datos sobre los sujetos que escriben en la universidad, pero muy pocos sobre el análisis de los textos escritos por los universitarios.

Por otro lado, en el período estudiado, no contamos con datos sobre percepción de autoeficacia de sujetos de países latinoamericanos hablantes de español, como es el caso de Chile. Ello podría deberse a que, en este país, y en el contexto latinoamericano en general, la inversión en investigación y en educación es menor que en países como Estados Unidos, Bélgica o Reino Unido, entre otros (OCDE, 2018), donde sí existe investigación sobre el tema. Además, si bien la escritura académica universitaria está siendo considerada como una nueva línea de investigación, puesto que es objeto de estudio y de enseñanza hace dos décadas en el contexto anglosajón y una década en el ámbito latinoamericano, aún son escasas las investigaciones sistemáticas sobre el tema (Salazar, 2015). 
Por ello, probablemente, la consideración de factores sociocognitivos (emoción, motivación, autopercepción, metacognición, etc.) en la investigación en escritura aún no han sido considerados o solo se ha hecho recientemente y de forma tangencial. Así, el estudio de la escritura en la universidad en Latinoamérica no se ha abordado de manera integral, ya que se ha focalizado en aspectos particulares (cognitivos, textuales, disciplinares) que no han sido interrelacionados, por lo que no se ha dado cuenta de la complejidad de la escritura como un proceso multidimensional. Por otro lado, en términos prácticos, la escritura es utilizada, generalmente, como un instrumento de reproducción de información, esto es, como una herramienta de comunicación de lo que se sabe o de lo que se piensa sobre un tema determinado, más que como un instrumento de reflexión sobre las propias capacidades de las personas. Ello podría explicarla ausencia de investigación en Latinoamérica sobre la relación de la escritura con otras variables de tipo cognitivo o metacognitivo.

En síntesis, hasta ahora, son escasísimas, o, en algunas latitudes, inexistentes, las investigaciones que vinculen la autoeficacia en la escritura y su relación con el desempeño escrito. Sin embargo, en Chile, encontramos algunos avances en un área similar, que dice relación con las concepciones sobre la escritura y su relación con el rendimiento escrito, tanto en estudiantes de educación básica (Aguilar et al., 2016) como en aquellos de programas de formación inicial docente (Errázuriz, 2017).

Finalmente, si bien el objetivo de este trabajo no es aportar nuevas evidencias o métodos sobre el tema en cuestión en Chile, sí ofrece una sistematización útil y necesaria acerca de los instrumentos sobre autoeficacia en escritura académica, sus validaciones y resultados. En este sentido, el aporte del artículo radica en impulsar el estudio de la autoeficacia en la escritura académica, línea de investigación que podría proporcionar nuevos datos e insumos para abordar el problema y, así, diseñar medidas pertinentes para aportar en su resolución. Esto, ya que la gran mayoría de las investigaciones en Chile y Latinoamérica se han enfocado en el desempeño escrito (Uribe y Carrillo, 2001; Sabaj, 2009; Sotomayor et al., 2017, entre muchos otros), en lugar de intentar comprender cómo los estudiantes conceptualizan la escritura académica y sus propias capacidades para esta labor en las distintas disciplinas.

\section{CONCLUSIONES}

El propósito de este estudio fue hacer una revisión crítica de los diferentes instrumentos para medir la autoeficacia asociada a la competencia para la escritura académica: sus características técnicas y psicométricas, y los principales resultados de esas investigaciones. De acuerdo con los resultados de la investigación, se puede concluir que: 1. La mayoría de los estudios utilizaron instrumentos sobre autoeficacia asociada a la producción de textos, aunque también en algunos casos se incluyó, además, la autoeficacia para la lectura. 2. El constructo de autoeficacia está bien definido y no presenta ambigüedad, pero no ocurre lo mismo con la producción de textos, ya que las dimensiones consideradas son muy heterogéneas entre los diferentes estudios. Además, no se considera el hecho de que la eficacia para la producción de textos puede depender del tipo de texto, el que puede ser muy diferente dependiendo de la especialidad de pregrado que se curse. 3. El 62,5\% de de los estudios analizados no reportan procedimientos para validar las mediciones o reportan Análisis de Componentes Principales, por lo que no estarían cumpliendo con los actuales estándares para la validación de instrumentos. No obstante, quienes sí lo hicieron mostraron indicadores adecuados. 4. La percepción de autoeficacia para la escritura se ha estudiado de forma homogénea, lo que se evidencia en que se han mantenido las características centrales de los instrumentos seminales sobre percepción de autoeficacia: se presentan principalmente en inglés, se desarrollan mayoritariamente en Estados Unidos y se construyen a partir de oraciones declarativas en primera persona. 5. En conclusión, se requiere estudiar de forma más rigurosa la relación entre la autoeficacia para la producción de textos académicos y el desempeño objetivo de los estudiantes en la producción de textos, especialmente, considerando textos propios de disciplinas altamente especializadas.

\section{AGRADECIMIENTOS}

La investigación fue realizada gracias al financiamiento del Proyecto FONDECYT de Iniciación N11170128, titulado "Caracterización discursiva de géneros producidos por estudiantes de Derecho y Medicina: su relación con la percepción de la autoeficacia en la escritura, la calidad general del texto y la evaluación disciplinar"; y al apoyo del Instituto de Investigación Multidisciplinar en Ciencia y Tecnología de la Universidad de La Serena.

\section{REFERENCIAS}

Aguilar, P., P. Albarrán, M. Errázuriz y C. Lagos, Teorías implícitas sobre los procesos de escritura: Relación de las concepciones de estudiantes de Pedagogía Básica con la calidad de sus textos, doi: 10.4067/S071807052016000400001, Estudios pedagógicos, 4(3), 7-26 (2016).

Andrade, H., X. Wang, Y. Du y R. Akawi, Rubric-referenced self-assessment and Self-efficacy for writing, doi: 10.3200/JOER.102.4.287-302, The Journal of Educational Research, 102(4), 287-302 (2009). 
Badley, G., Academic writing as shaping and re-shaping, doi: 10.1080/13562510902757294, Teaching in Higher Education, 14(2), 209-219 (2009).

Bandura, A., Self-efficacy: toward a unifying theory of behavioral change, doi: 10.1037/0033-295X.84.2.191, Psychological review, 84(2), 191-215 (1977).

Bandura, A., Self-efficacy mechanism in human agency, doi: 10.1037/0003-066X.37.2.122, American Psychologist, 2, 122147 (1982).

Bandura, A., B. Taylor y otros tres autores, Catecholamine secretion as a function of perceived coping self-efficacy, doi: 10.1037/0022-006X.53.3.406, Journal of Consulting and Clinical Psychology, 53(3), 406-414 (1985).

Bandura, A., Perceived self-efficacy in cognitive development and functioning, doi: 10.1207/s15326985ep2802_3, Educational Psychologist, 28(2), 117-148 (1993).

Bandura, A., Self-efficacy: The exercise of control, 14ª Ed., 1-604, Freeman, New York, Unites States (1997).

Bandura, A., Guide to constructing self-efficacy scales; in Self-efficacy beliefs of adolescents by F. Pajares y T. Urdan, $1^{\mathrm{a}}$ Ed., Information age Publishing, pp. 307-337, Greenwich, Unites States (2006).

Bandura, A., Social cognitive theory; in Handbook of Theories of Social Psychology by Van Lange P.M., A.W. Kruglanski and E. Higgins, $1^{\text {a }}$ Ed., Sage Publications Ltd., Vol.1, pp. 349-373, Thousand Oaks, Unites States (2012).

Bassi, J., La escritura académica: 14 recomendaciones prácticas, doi: 10.5565/rev/athenea.1986, Athenea Digital, 17(2), 95-147 (2017).

Bitrán, M., D. Zúñiga y otros tres autores, Mejoría en las habilidades de comunicación escrita de estudiantes de medicina: Impacto de un taller de escritura, doi: 10.4067/S0034-98872009000500004, Revista Médica de Chile, 137(5), 617-624 (2009).

Blanco, A., Creencias de autoeficacia en estudiantes universitarios: un estudio empírico sobre la especificidad del constructo, doi: 10.7203/relieve.16.1.4149, Relieve, 16(1), 1-28 (2010).

Blanco, H., J. Aguirre, J. Barron y J. Blanco, Composición Factorial de la Escala de Autoeficacia Académica en Universitarios Mexicanos, doi: 10.4067/S0718-50062016000200009, Formación Universitaria, 9(2), 81-88 (2016).

Boscolo, P. y S. Hidi, The Multiple Meanings of Motivation to Write; in Writing and Motivation by Hidi, S. and P. Boscolo, 1st Ed., Elsiever, pp. 1-14, Oxford, England (2007).

Bruning, R., M. Dempsey y otros tres autores, Examining Dimensions of Self-Efficacy for Writing, Journal of Educational Psychology, doi: 10.1037/a0029692, 105(1), 25-38 (2013).

Büyükikiz, K., Y. Uyar y A. Balci, A writing self-efficacy scale for non-native students of Turkish origin: $A$ validity and reliability study. International Online Journal of Educational Sciences, 5(2), 302-313 (2013).

Carlino, P., Escribir, leer, y aprender en la universidad. Una introducción a la alfabetización académica, 1르. Ed., 9-185, Fondo de Cultura Económica, Buenos Aires, Argentina (2005).

Castells, N., M. Mateos y otros tres autores, Profiles of self-perceived competencies and conceptions of academic writing in university students, doi: 10.1080/11356405.2015.1072358, Culture and Education, 27(3), 569-593 (2015).

Corry, M. y J. Stella, Teacher self-efficacy in online education: a review of the literature, doi: 10.25304/rlt.v26.2047, Research in Learning Technology, 26(1), 1-12 (2018).

De Smul, M., S. Heirweg y otros tres autores, How competent do teachers feel instructing self-regulated learning strategies? Development and validation of the teacher self-efficacy scale to implement self-regulated learning, doi: 10.1016/j.tate.2018.01.001, Teaching and Teaching Education 71, 214-225 (2018).

Demir, R., The relation between the Awareness Level of Writing Strategies and Writing Self-Efficacy, doi: 10.5539/ies.v11n5p59, International Education Studies, 11(5), 59-73 (2018).

Ekholm, E., S. Zumbrunn y S. Conklin, The relation of college student self-efficacy toward writing and writing self-regulation aptitude: writing feedback perceptions as mediating variable, doi: 10.1080/13562517.2014.974026, Teaching in Higher Education, 20(2), 197-207 (2014).

Errázuriz, M., Teorías implícitas sobre la escritura académica en estudiantes de programas de formación inicial docente: ¿inciden en el desempeño escrito?, doi: 10.11144/Javeriana.syp36-71.tiea, Signo y Pensamiento, 36(71), 36-52 (2017).

Golombek, C., K. Klingsieck e I. Scharlau, Assessing Self-Efficacy for Self-Regulation of Academic Writing Development and Validation of a Scale, doi: 10.1027/1015-5759/a000452, Journal of Pshychological Assessment, 2(1),1-11 (2018).

Hashemnejad, F., M. Zogui y D. Amini, The relationship between Self-efficacy and Writing Performance across Genders, doi: 10.4304/tpls.4.5.1045-1052, Theory and Practice in Language Studies, 4(5) 1045-1052 (2014).

Honicke, T. y J. Broadbent, The influence of academic self-efficacy on academic performance: A systematic review, doi:10.1016/j.edurev.2015.11.002, Educational Research Review, 17(1), 63-84 (2016).

Irving, L., What is "academic writing"?; in Writing Spaces: Readings on writing by Ch. Lowe y P. Zamliansky, $1^{\mathrm{a}}$ Ed., Parlor Press LLC, Vol 1, pp. 3-17, West Lafayette, Unites States (2010). 
Jackson, S., Research Methods and Statistics: A Critical Thinking Approach, $3^{\mathrm{a}}$ Ed., 20-427, Wadsworth Publishing Group, Belmont, United States (2009).

Jones, L., J. Varberg y otros tres autores, Reading and writing self-efficacy of incarcerated adults, doi: 10.1016/j.lindif.2012.01.008, Learning and Individual Differences, 22, 343-349 (2012).

Kavanoz, S. y G. Yüksel, Developing and Validating a Self-Efficacy Scale for Scholarly Writing in English, doi: 10.15345/iojes.2016.02.007, International Online Journal of Educational Sciences, 8(2), 71-82 (2016).

Khojasteh, L., N. Shokrpour y M. Afrasiabi, Relationship between Writing Self-efficacy Performance of Iranian EFL Students, doi: 10.7575/aiac.ijalel.v.5n.4p.29, International Journal of Applied Linguistics \& English Literature, 5(4), 29-37 (2016).

Klassen, R. y J. Klassen, Self-efficacy beliefs of medical students : A review of the research, doi: 10.1007/s40037-0180411-3, Perspectives on Medical Education, 7(1), 76-82 (2018).

Kruger, J. y D. Dunning, Unskilled and unaware of it: How difficulties in recognizing one's own incompetence lead to inflated self-assessments, doi: 10.1037/0022-3514.77.6.1121, Journal of Personality and Social Psychology, 77, 1121-1134 (1999).

Lichtinger, E., Gap Between Self-efficacy and College Students' Writing Skills, doi: 10.1080/10790195.2017.1411213, Journal of College Reading and Learning, 48(2), 124-137 (2018).

Lloret-Segura, S., A. Ferreres-Traver, A. Hernández-Baeza y I. Tomás-Marco, El análisis factorial exploratorio de los items: una guía práctica, revisada y actualizada, doi: 10.6018/analesps.30.3.199361, Anales de Psicología, 30(3), 1151-1169 (2014).

Lowe, C., y P. Zemliansky, Writing Spaces: Readings on Writings, $1^{\mathrm{a} E d .,}$ vol.1, Parloss Press LLC, Anderson, Unites States (2010).

Luszczynska, A., F. Gibbons, B. Piko y M. Tekozel, Self-regulatory cognitions, social comparison, perceived peers' behaviors as predictors of nutrition and physical activity: A comparison among adolescents in Hungary, Poland, Turkey, and USA, doi: 10.1080/0887044042000205844, Psychology and Health, 19, 577-593 (2004).

Martins, R., C. Pimentel y M. Costa, Construção e validação da Escala Unifatorial de Autoeficácia Acadêmica, doi: 10.5935/2175-3520.20170002, Psicologia da Educação, 44(1), 15-23 (2017).

McCarthy, P., S. Meier y R. Rinderer, Self-efficacy and writing: A different view of self-evaluation, doi: 10.2307/357865, College Composition and Communication, 36(1), 465-471 (1985).

Muñiz, J. y M. Fonseca-Pedreros, Diez pasos para la construcción de un test, doi: 10.7334/psicothema2018.291, Psicothema, 31(1), 7-16 (2019).

OCDE, La OCDE sostiene que es necesario redoblar los esfuerzos para mejorar la equidad en la educación (2018).

Olivari, C. y E. Urra, Autoeficacia y conductas de salud, doi: 10.4067/S0717-95532007000100002, Ciencia y Enfermería, 13(1), 19-15 (2007).

Pajares, F., Self-efficacy beliefs, motivation, and achievement in writing: $A$ review of literature, doi: 10.1080/10573560308222, Reading and Writing Quarterly, 19(1), 139-158 (2003).

Pajares, F., Empirical properties of a scale to assess writing self-efficacy in school contexts, doi: 10.1080/07481756.2007.11909801, Measurement and Evaluation in Counseling and Development, 39(1) 239-249 (2007).

Pajares, F. y G. Valiante, Self-efficacy beliefs and motivation in writing development; in Handbook of writing research by MacArthur, C.A., S. Graham y J. Fitzgerald, $1^{\text {a }}$ Ed., Guilford Press, pp. 158-170. New York, Unites States (2006).

Pajares, F., J. Hartley y G. Valiante, Response format in writing self-efficacy assessment: Greater discrimination increases prediction, Measurement and Evaluation in Counseling and Development, 33(1), 214-221 (2001).

Pajares, F., M. Miller y M. Johnson, Gender differences in writing self-beliefs of elementary school students, doi: 10.1037/0022-0663.91.1.50, Journal of Educational Psychology, 91(1), 50-61 (1999).

Pak-Tao, P., Effective writing: a social science student, $1^{\text {a }}$ Ed., The Chinese University Press, Hong Kong, China (2008).

Podsakoff, P., S. MacKenzie y N. Podsakoff, Sources of method bias in social science research and recommendations on how to control it, doi: 10.1146/annurev-psych-120710-100452, Annual Review of Psychology, 63(1), 539-569 (2012).

Prat-Sala, M. y P. Redford, The interplay between motivation, self-efficacy and approaches to studying, doi: 10.1348/000709909X480563, British Journal of Educational Psychology, 80, 283-305 (2010).

Prat-Sala, M. y P. Redford, Writing essays: Does self-efficacy matter? The relationship between self-efficacy in reading and in writing and undergraduate students' performance in essay writing, doi: 10.1080/01443410.2011.621411, Educational Psychology, 31(1), 9-20 (2012).

Prickel, D., The development and validation of a writing self-efficacy scale for adult basic writers and its use in correlational analysis, doctoral thesis, Oregon State University, Oregon, Estados Unidos (1994).

Ramos-Villagrasa, P., I. Sanchez-Iglesias y otros 4 autores, Version of "Self-efficacy for Writing Scale" (SEWS), doi: 10.6018/analesps.34.1.264931, Anales de Psicología, 34(1), 86-91 (2018). 
Rodríguez, A., J. Espinoza, L. Ramírez y A. Ganga, Deserción Universitaria: Nuevo Análisis Metodológico, doi: 10.4067/S0718-50062018000600107, Formación Universitaria, 11(6), 107-118 (2018).

Rubin, A. y E. Babbie, Research Methods for Social Work. 7ª Ed.,599-653, Brooks Cole, Belmont, Unites States (2011).

Sabaj, O., Descubriendo algunos problemas en la redacción de Artículos de Investigación Científica (AIC) de alumnos de postgrado, doi: 10.4067/S0718-09342009000100006, Revista Signos. Estudios de Lingüística, 42(69), 107-127 (2009).

Salazar, C., La escritura académica universitaria: diferentes perspectivas de estudio, doi: 10.32870/dse.v0i11.275, Diálogos sobre educación. Temas actuales en investigación educativa, 6(11), 1-21 (2015).

Salinas, A., E. Chandía y D. Rojas, Validación de un instrumento cuantitativo para medir la práctica reflexiva de docentes en formación, doi: 10.4067/S0718-07052017000100017, Estudios pedagógicos, 43(1), 289-309 (2017).

Sanders-Reio, J. Investigation of the relations between domain-specific beliefs about writing, writing self-efficacy, writing apprehension, and writing performance in undergraduates, doctoral thesis, University of Maryland, Washington, Estados Unidos (2010).

Sanders-Reio, J., P. Alexander, T. Reio y I. Newman, Do students' beliefs about writing relate to their writing self-efficacy, apprehension, and performance?, doi: 10.1016/j.learninstruc.2014.02.001, Learning and Instruction, 33, 1-11 (2014).

Schmidt, K. y J. Alexander, The empirical Development of an Instrument to Measure Writerly Self-efficacy in writing centers, The journal of Writing Assessment, 5(1), 1-10 (2012).

Shell, D., C. Murphy y R. Bruning, Self-efficacy and outcome expectancy mechanisms in reading and writing achievement, doi: 10.1037/0022-0663.81.1.91, Journal of Educational Psychology, 81(1), 91-100 (1989).

Shell, D., C. Colvin y R. Bruning, Developmental and ability differences in self-efficacy, causal attribution, and outcome expectancy mechanisms in reading and writing achievement, doi: 10.1037/0022-0663.87.3.386, Journal of Educational Psychology, 87(1), 386-398 (1995).

Sherer, M., J. Maddux y otros cuatro autores, The self-efficacy Scale: Construction and validation, doi: 10.2466/pr0.1982.51.2.663, Psychological Report, 51(1), 663-671 (1982).

Sotomayor, C., N. Ávila y otros cuatro autores, Desempeño ortográfico de estudiantes chilenos: claves para la enseñanza de la ortografía, doi: 10.4067/S0718-07052017000200017, Estudios pedagógicos, 43(2), 315-332 (2017).

Staples, S., J. Egbert, D. Biber y B. Gray, Academic Writing Development at the University Level: Phrasal and Clausal Complexity Across Level of Study, doi: 10.1177/0741088316631527, Discipline, and Genre, 33(2), 149-183 (2016).

Tapia, M., G. Burdiles y B. Arancibia, Aplicación de una pauta diseñada para evaluar informes académicos universitarios, doi: 10.4067/S0718-09342003005400009, Revista Signos, 36(54), 249-257 (2003).

Tardy, C., Writing for the World: Wikipedia as a introduction to academic writing, English Teaching Forum, 48(1), 1-12 (2010).

Uribe, O. y S. Carrillo, Relación entre la lecto-escritura, el desempeño académico y la deserción estudiantil, Entramado, 10(2), 272-285 (2014).

Villalón, R., M. Mateos y I. Cuevas, High school boys's and girls's writing conceptions and writing self-efficacy beliefs: What is their role in writing performance?, doi: 10.1080/01443410.2013.836157, Educational Psychology, 35(6) 653-674 (2013).

Willits, F., G. Theodori, y A. Luloff, Another look at Likert scales, Journal of Rural Social Sciences, 31(3), 126-139 (2016).

Wyatt, M., Language Teachers' Self-efficacy Beliefs: A Review of the Literature (2005-2016), doi: 10.14221/ ajte.2018v43n4.6, Australian Journal of Teacher Education, 43(4), 92-120 (2018).

Yokoyama, S., Academic Self-Efficacy and Academic Performance in Online Learning: A Mini Review, doi: 10.3389/fpsyg.2018.02794, Frontiers in Psychology, 9(1), 27-94 (2019).

Zimmerman, B. y A. Bandura, Impact of self-regulatory influences on writing course attainment, doi: 10.3102/00028312031004845, American Educational Research Journal, 31(1), 845-862 (1994).

Zimmerman, B., y A. Kitsantas, Reliability and validity of Self-Efficacy for Learning Form (SELF) scores of college students, doi: 10.1027/0044-3409.215.3.157, Journal of Psychology, 215(3), 157-163 (2007). 